\title{
The ANTARES telescope turns its gaze to the sky
}

\author{
T. Stolarczyk, \\ CEA Saclay, DSM/Dapnia/SPP • France
}

$\mathrm{O}$ n Thursday, 2 March 2006, the first detection line of the ANTARES neutrino telescope, which lies $2,500 \mathrm{~m}$ below the surface of the sea, was connected to the shore station in La Seyne-sur-Mer by a remote-controlled robot. A few hours later, ANTARES looked up at the sky for the first time and caught sight of its first muons.

This connection marks the arrival of the ANTARES detector, the first deep-sea, high-energy neutrinotelescopein the Mediterranean. The event rewards ten years of combined efforts by some 200 technicians, engineers and scientists from twenty laboratories (France, Germany, Italy,Spain, The Netherlandsand Russia).

ANTARES is located about 30 kilometres off the French coast near Toulon. The detector when completed will consist of 12 vertical strings supporting 900 photomultipliers in total. The measurement of the Cerenkov light emitted by muons produced in muon-neutrino interactions in water and under-sea rock will allow the reconstruction of the neutrino direction with an accuracy of 0.3 degree above $10 \mathrm{TeV}$, thus justifying the term 'neutrino astronomy'.

\section{Why a neutrino astronomy?}

The sky has been observed from antiquity with photons. The second half of the last century has seen the birth of astronomy outside the visible light domain and in particular $\gamma$ ray astronomy (E > $10 \mathrm{MeV}$ ). Each time a new wavelength has been used, unexpected phenomena were observed. Our view of the Universe today reveals objects such as supernova remnants, pulsars, micro-quasars in the Galaxy, extragalactic y ray bursts and active galactic nuclei, all emitters of very high energy photons (see Fig. 1).

Tremendous progress has been made during the last century in understanding these new objects, helped in that by the fact that many of them are detected in several wavelength domains and in particular at higher and higher energies. However, photon observations have their limitations. Because of their interaction with the infrared or cosmological diffuse backgrounds or with intergalactic matter, they cannot travel more than $10 \mathrm{Mpc}$ above $10^{14} \mathrm{eV}$ (The visible universe is $-5 \mathrm{Gpc}$, where $1 \mathrm{pc}-3.2$ light year $-310^{13} \mathrm{~km}$ ).

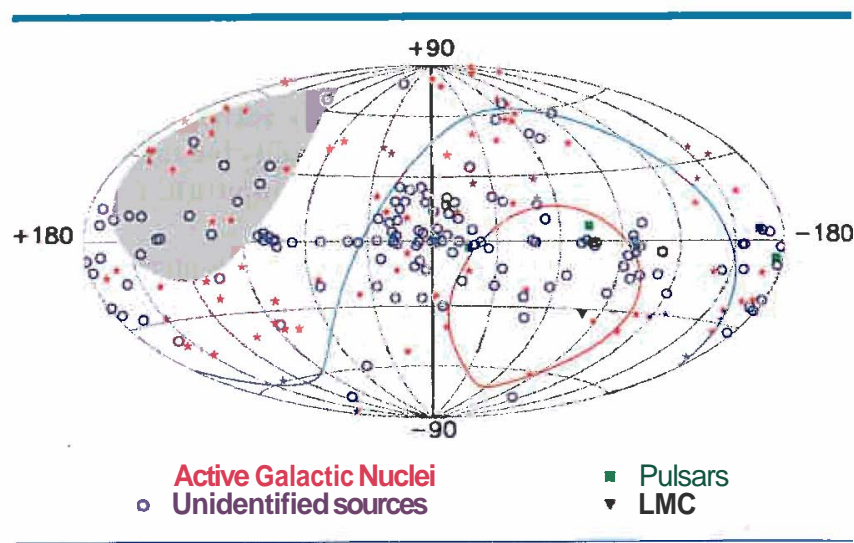

A Fig. 1: High energy $\gamma$ ray sources $(E>100 \mathrm{MeV})$ from the $3^{\text {rd }}$ Egret catalogue (Instrumentoperated on the CGRO satellite between 1991 and 1995). This map presents 271 sources in total among which 170 are not identified. Only the grey area is never seen by a neutrino detector in the Mediterranean. Most of these sources may emit high energy neutrinos. The source concentration in the middle of the map corresponds to the galactic Centre.
Another view of the sky is given by cosmic rays. This is particularly true with the new generation of ground observatories that will detect particles up to $10^{20} \mathrm{eV}$. They could help to elucidate the origin of cosmic rays on which scientists have been debating for tens of years. However, here again, the fact that protons are subject to interaction with diffuse photon backgrounds on their path to the detector limits the observation depth to 50 $\mathrm{Mpc}$ at $10^{20} \mathrm{eV}$. Moreover, being deviated by intergalactic magnetic fields, they are of little help in point-source searches at lower energies.

The neutrino appears to be an excellent candidatefor high energy astronomy: it is neutral and insensitive to magnetic fields. It essentially does not interact with matter. It makes the Universe totally transparent over a wide energy range. As an example, although from a lower energy domain, photons produced in the core of the Sun during fusion reactions become visible light and reach the Sun outer layers after one million years. On the other hand, neutrinos that are produced in the same reactions escape immediatelyfrom the star and reach the Earth after 8 minutes. This example shows how neutrinos can give unique information on the core of a source.

High energetic photons are usually interpreted as being the result of electron acceleration. High energy neutrinos should be produced from the interaction of accelerated protons on the surrounding matter $\left(\mathrm{p}+\mathrm{A} / \gamma_{\mathrm{amb}} \rightarrow \pi^{\circ}+\pi^{ \pm}+\ldots\right.$ followed by $\pi^{ \pm} \rightarrow \mu \mathrm{v}$, and $\mu \rightarrow \nu_{\mu} v_{e} e$, where A represents the surrounding matter and $\gamma_{a m b}$ the ambient low energy photon field). Under these assumptions, part of the high energy photon flux could also be due to proton interactions from the $\mathrm{n}^{\mathrm{o}} \rightarrow \mathrm{y}+\gamma$ decays.

Possible sources of high energy neutrinos in the Galaxy are binary stars with a neutron star or a black hole accreting matter from its companion. The accretion process creates plasma waves in the strong magnetic field of the compact object, leading to stochastic acceleration at high energies. The interaction of these particles with the accreting matter or the companion star would then produce high energy neutrinos.

Explosions of massive stars in the Galaxy (supernovae)produce an expanding shell of matter which is known from radio observations to accelerate high energy particles. Protons inside supernova shells can be accelerated by a first order Fermi mechanism (diffusion of charged particles on magnetic field inhomogeneities inside moving plasmas). The interaction of these protons with the shell gives neutrinos.

Active galactic nuclei are the most powerful objects observed in the Universe. They are galaxies hosting a super massive black hole $\left(10^{6}\right.$ to $10^{10}$ solar masses) accreting matter at a rate of a few solar masses peryear. Part of the absorbed energy is then released in a jet of accelerating electrons, these electrons being further able to give energy to ambient photons (the inverse Compton process). Electrons are accelerated by the Fermi process that should apply to $\mathrm{p}^{\text {rotons }}$

Gamma ray bursts are the most violent phenomena in the Universe. The energy released in the few tens of seconds of the emission is estimated to be up to $10^{45} \mathrm{~J}$. A possible interpretation of such an event is that a compact object emits matter that further expands relativistically in the surrounding medium. This would naturally produce high energy neutrinos. 
Up to now no high energy neutrino source has been discovered.Detectingneutrinos from already known sources would help to understand better the origin of the high energy phenomena. Because neutrinos make the universe transparent, sources with no photonic counterpart could also be discovered. The discovery of such neutrinosources would imply the presence of proton acceleration and help in answering the long standing question of the origin of the high energy cosmic rays.

\section{Detection principle}

Their very weak interaction with matter makes neutrinos hard to detect. This problem can only be overcome by using the largest detectors, shielded against the cosmic radiation which constantly bombards all sites on the Earth and generates significant and continuous background noise. Neutrino telescopes are mainly sensitive to the $1 \mathrm{TeV}-1000 \mathrm{TeV}$ energy range.

The principle of neutrino telescopes is based on the detection of the very faint trails of light that upward going muons leave behind in a large instrumented volume of water or ice. These upward going muons result from the interaction of neutrino-muons with the Earth's crust. The typical path of a high energy muon in the rock is a few kilometres so that even a relatively modest sensitive volume ( $100 \mathrm{~m}$ being a typical scale) can indirectly detect the neutrinos from the much larger interaction volume (of a $10 \mathrm{~km}$ scale) below the detector. The phenomenon can be observed because of the total darkness at these abyssal depths.

The ANTARES detector, being placed at $43^{\circ} \mathrm{N}$ in the Mediterranean, observes the sky in the southern hemisphere through the Earth (see Figure 1). It covers a complementary region compared to the South Pole neutrino detector IceCube [1]. In particularit includes the Galactic Centre, a region that reveals high energy phenomena as recently shown by the Hess high-energy $\gamma$ observatory [2].

ANTARES could also give information on the dark matter problem. First revealed 70 years ago, this problem is now a central question in cosmology.Most of the content of the Universe - 95\% of its mass - is still mysterious: it is expected to consist of at least two components called dark matter and dark energy. Dark matter might be composed of particles known as weakly interacting massive particles or "wimps". The physics theory referred to as supersymmetrypredicts their existence, but their demonstration is now needed. According to this theory, wimps, which can be both particle and anti-particle,would eventually annihilate, producing jets that include neutrinos.ANTARES could observe the low-energy neutrinos $(10$ to $100 \mathrm{GeV})$ produced from the annihilation of wimps gravitationallytrapped at the centre of the Earth, the Sun or the Galaxy.

The interaction of cosmic rays in the atmospherecreates showers of particles among which only muons and neutrinos are penetratingenough to reach the detector. The downward atmospheric muon flux is a potential background although reduced by order of magnitudes by the water above the detector. Interactions of atmospheric neutrinos coming from above give a negligiblecontribution to the downward muon flux. By contrast, those produced at the antipodes mimic the cosmic signal. Fortunately they have a harder spectrum and they become rapidly negligible as their energy increases, usually above 10 Tell

An extra background is the continuous faint light coming from two distinct origins: firstly the Cerenkov light of $\mathrm{MeV}$ electrons resulting from ${ }^{40} \mathrm{~K}$ beta-decay in the sea water; secondly the contribution from the bioluminescent abyssal fauna.

ANTARES is also a multidisciplinary underwater-science infrastructure continuously recording various types of data for studies relating to oceanography - including observation of the

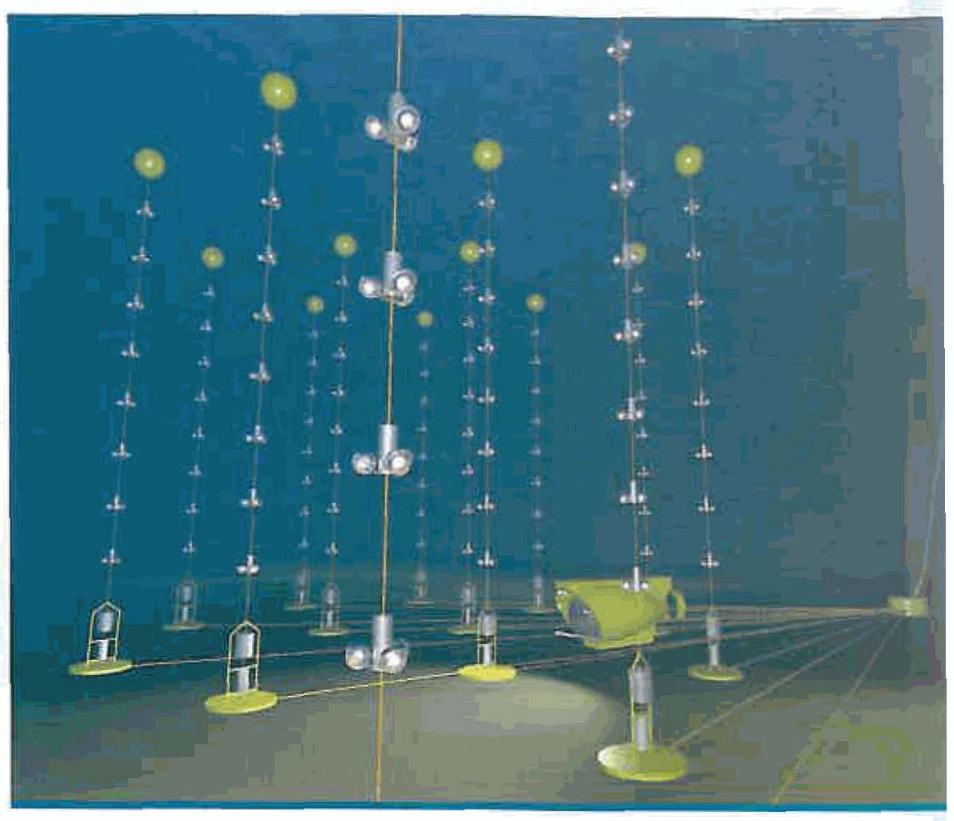

A Fig. 2: Artist's impression of the ANTARES neutrino telescope on the sea floor, showing the detector lines, the seabed interlinkcables, the junction box and the cable to the shore. In this illustration for clarity, the number of storeys per line is reduced and many items are not drawn to scale (C F. Montanet, CNRS/IN2P3 and UFJ).

deep marine environment and bioluminescent phenomena - and geophysics. One example of this is the seismographinstalled there, which has been recording Earth tremors for the past year.

\section{The detector}

An artist's impression of the layout of the ANTARES neutrino telescope is shown in Figure 2. The detector consists of 12 lines spread over a $200 \times 200 \mathrm{~m}^{2}$ area and spaced by around 65 metres, at a depth of 2500 metres. Each line has a total height of $-450 \mathrm{~m}$. They are weighted to the sea bed and held nearly vertical by a buoy at the top. A line has a total of 25 storeys each comprising 3 optical modules. They consist of 10 inch photomultipliershoused in glass spheres that detect Cherenkov light. They are oriented $45^{\circ}$ downward to increase the sensitivity to upward going tracks [3][4].The first storey is 100 metres above the sea bed, and the spacing between storeys is 14.5 metres. Each storey comprises a titanium electronic container where the analogue electrical outputs of the photomultipliers are digitised in a custom built microchip. The signal is then treated in real time by a data acquisition card. The result is finally sent to the bottom of the line through optical fibres.

Each line anchor is connected to a junction box on the seabed via an electro-optical cable. In the junction box the outputs from up to 16 lines are gathered onto a 40 kilometre electro-optical submarine cable and sent to the experiment shore station in the town of La Seyne-sur-Mer,in France. Since no filteringis made offshore, the data stream is mainly composed of background hits. On shore, a computer farm selects from the data the periods of time containing storeys in coincidence, suspected to contain physical tracks. These events are stored on disks for further selections and analysis. The knowledge of the fired photomultiplier positions and the arrival time of the Cerenkov photons allow the reconstruction of the muon track thus giving information on the parent neutrino.

The lines are flexible and so move in the sea current, with movements being 5 metres at the top for a typical sea current of $5 \mathrm{~cm} / \mathrm{s}$. - 


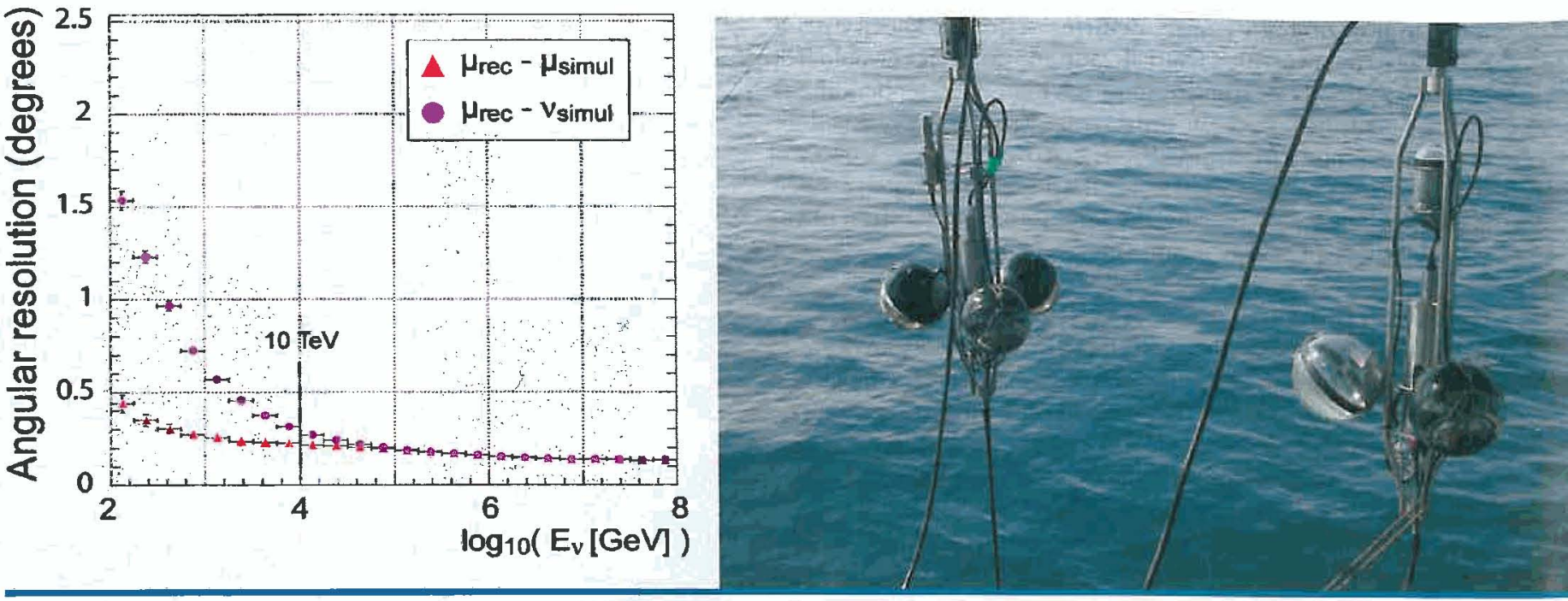

A Fig. 3: Left: Angular resolution of the ANTARES detector as a function of the energy of the incident neutrino

Right: picture of the second storey of the mini-instrumented line during its deployment in March 2005 (C) ANTARES collaboration).

.. The positions of the optical modules are measured with an acoustic positioning system with components at discrete positions on the line and on the sea bed, together with tiltmeters and compasses on each storey of the line. The positioning system gives a real time measurement, typically once every few minutes, of the position of every optical measurementwith a spatial precision better than $10 \mathrm{~cm}$.

During the extensive trials carried out in the R\&D phase (1996-1999),the properties of the selected site have been carefully studied.Absorption and scattering lengths are of about 60 and 300 metres in the blue wavelength, and of about 26 and 100 metres in the UV wavelength, respectively [5]. The loss in transmission due to biological fouling and sedimentation deposits on the surface of the optical modules is less than $1.5 \%$ per year [6] The optical background was also monitored. The 10 inch ANTARES photomultipliers have a continuous rate of 60 to $100 \mathrm{kHz}$ on top of which some short bursts are due to episodic fauna activity [7].

\section{Measured and expected performances}

In March 2005 a so-called mini-instrumentedline equipped with three storeys was deployed [8]. Its primary goal is to monitor the environmental parameters and to illuminate the full detector by means of several optical beacons. These beacons are able to emit flashes of light of a few nanoseconds in duration thus allowing the time calibration of the full detector. Moreover a seismometer is linked by a 50 metre cable to the instrumented anchor. Since its deployment, it has registered many earthquakes all round the world.

The anchor holds a laser beacon and an acoustic positioning receiver-transmitter.The bottom storey lies $100 \mathrm{~m}$ above the sea bed and is equipped with an optical beacon, a conductivity-temperature probe, an apparatus for light transparency monitoring and a hydrophone acting as an acoustic positioning receiver. The middle storey,located a further 15 metres above, comprises 3 optical modules and a sound velocimeter (see Figure 3, right). Finally the top storey, at a further 50 metres above, has another optical beacon and an acoustic Doppler current profiler.

The measurement of the sound velocity is important to convert time measurement with the acoustic positioningsystem into distances. The sound velocimeter records a value of about $1545 \mathrm{~m} / \mathrm{s}$ with a very good stability. The sound velocity depends on the tem- perature, pressure and conductivity which are independentlymonitored. The temperature on site is $13.2^{\circ} \mathrm{C}$ with variations of $0.1^{\circ} \mathrm{C}$ at most on a monthly scale.

This line allowed the measurement of some key performance figures in real conditions. In particular it was possible to check the timing precision of the apparatus at various stages. As an example the relative timing resolution of the photodetectors was obtained by illuminating the three optical modules on the middle storey with high amplitude signals from the optical beacon in the first storey. The distributions of the time differences between the three optical modules and the internal photomultipliers of the beacon, after subtraction of the light propagation offset, have a width of the order of $0.5 \mathrm{~ns}$.

A number of other specificationswere also shown to have been attained.For instance measurements of the distance between the bottom of the line and an emitter at 180 metres on the sea bed have shown a stability of a few centimetres.

These measurements show that the timing precision in ANTARES, for which the detector concept was designed, is only limited by the transit time spread in the optical modules (1.3 ns) and the light scattering in water. This shows experimentally that the ANTARES telescope should be able to reach a 0.3 " angular resolution above $10 \mathrm{TeV}$.

Figure 3, left shows the evolution of the angular resolution (the difference between the reconstructed and Monte Carlo generated angles) for muons and neutrinos versus the neutrino energy. At lower energy, the neutrino angular resolution deteriorates because the emitted muon is no longer collinear with the incident neutrino. Above $100 \mathrm{TeV}$ the resolution is better than 0.3 " for both muon and neutrino and is mainly limited by the light diffusion in water and the discrete energy loss of muons.

The event rate is related to the neutrino cross section and the efficiency to reconstruct muon tracks within a certain pointing accuracy.It can be quantified by the effective detector surface which is the ratio of detected muons per unit of time, divided by the incident neutrinoflux. It increasesWithenergy as the neutrino cross section does, and also because the muon range in the rock below the detector and the light produced by muons in water both increase with energy.However,because of the very small neutrino cross section, this area is very small: it reaches $1 \mathrm{~m}^{2}$ for vertical 

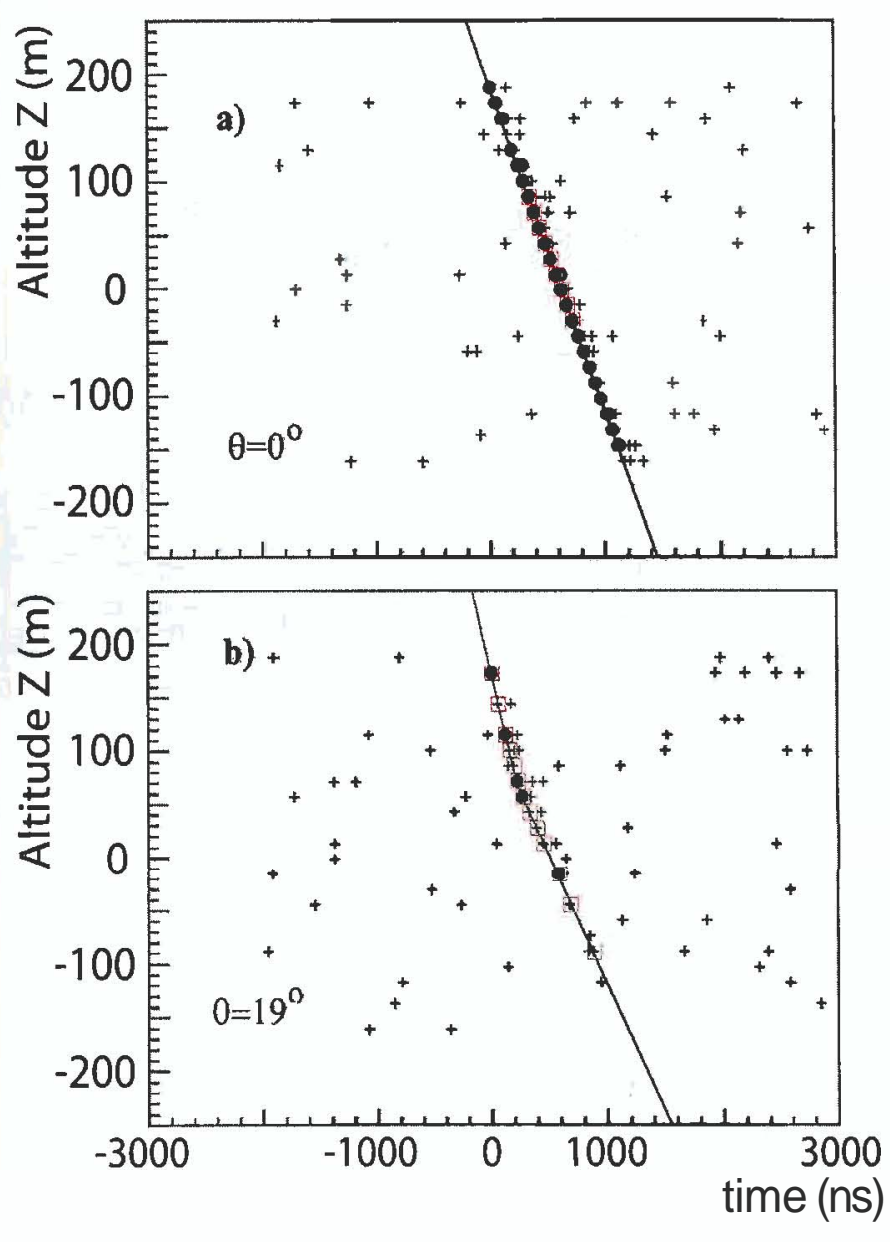

$\Delta$ Fig. 5: Examples of reconstructed tracks using the data from the first ANTARES line: a) vertical downward going muon; b) downward going muon at 19 degrees from the zenith.
V Fig. 6: The time differencesin the coincidence found between the optical beacon on the instrumented line and two storeys of the first detection line. Upper: storey at a higher altitude (distance of 150 metres). The Gaussian width is larger (2.6 ns) and the influence of the light scattering is responsiblefor the tail at larger times. Lower: storey at the same altitude (distance of 70 metres). The Gaussian width is $0.7 \mathrm{~ns}$.
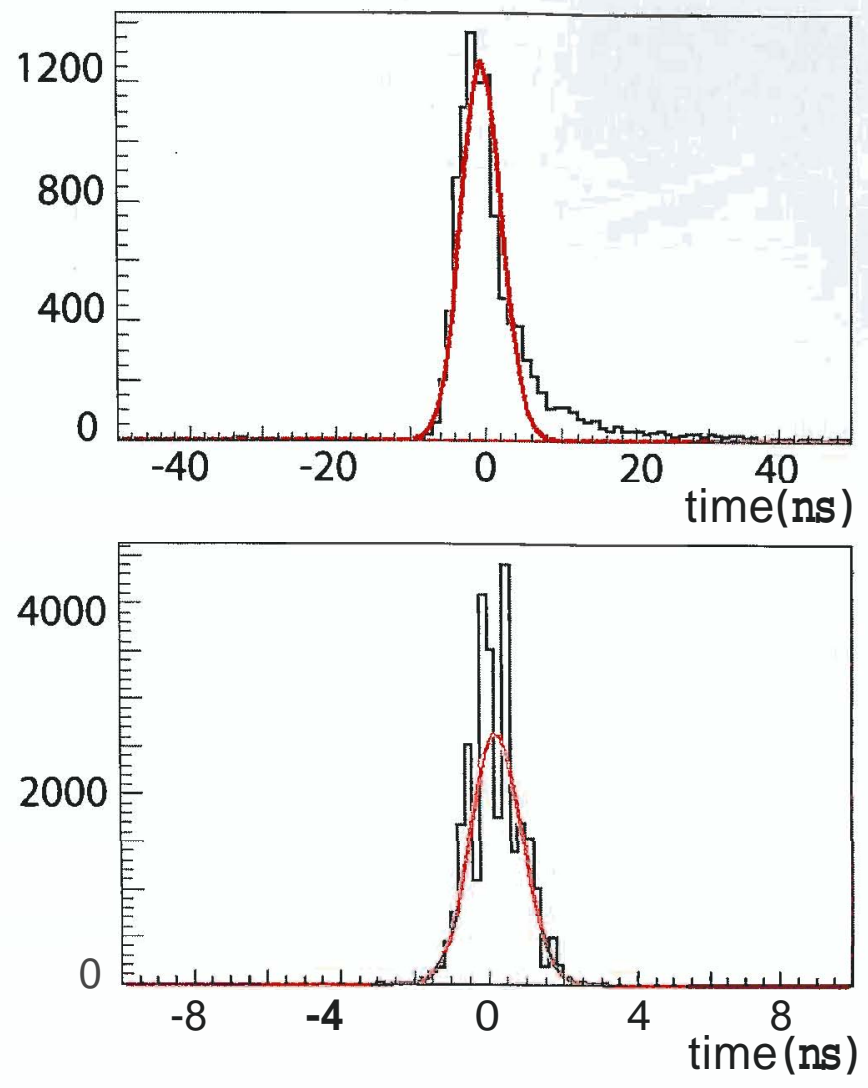

\section{What next?}

After ten years of continuousefforts, from site surveys to prototype deployments, the ANTARES collaboration has recently taken up the challenge of deploying a particle detector deep under the Mediterraneansea. The installation of the lines of the detector has now started with the deployment and operation of the first line since March, the deployment of the second line at the end of July and its connection on September $21^{\text {st }}$. Muons are now detected with both lines. Two more lines will be deployed by the end of the year, 4 more next spring, and the last 4 in autumn 2007. The operation of the detector is then planned for 5 years.

Beside the successful installation of ANTARES with many years of exciting results to come, the ANTARES collaboration is involved in the so-called KM3NeT design study ${ }^{1}$ to define the next kilometre-scale generation of neutrino telescoves in the Mediterranean. (More information on the experiment: http: llantares.in2p3.fr) $\%$

KM3NeT is a consortium of European laboratories among which are those involved in the ANTARES experiment and on two other projects NEMO and NESTOR. This consortium is funded by the European FP6 over the period 2006-2009 with the objective of producing a design study for a Mediterranean kilometre-cubeneutrino detector. More on www.krn3net.org.

\section{About the author:}

Thierry Stolarczyk received his PhD in 1990 for work on the Gallex experiment (solar neutrinos), then joined the NOMAD collaboration in 1994 (neutrino oscillations), and ANTARES in 1998. He is now Head of the CEA/Dapnia ANTARES and KM3NeT groups. From 1993 to 2001 he was a member of the French Physical Society Executive committee.

\section{References}

[1]IceCube, http:llicecube.wisc.edu.

[2] F. Aharonian et al,, Nature 439, 695-698, 2006.

[3] ANTARES collaboration, Nuclear Instruments and Methods in Physics Research A 555 (2005) 132-141

[4] ANTARES collaboration, Nuclear Instruments and Methods in Physics Research A 484 (2002) 369

[5] ANTARES collaboration, Astroparticle Physics 23 (2005) 131-155

[6] ANTARES collaboration, Astroparticle Physics 19 (2003) 253-267

[7] ANTARES collaboration, Astroparticle Physics 13 (2000) 127-136

[8] ANTARES collaboration, to appear in Astroparticle Physics, astro-phi0606229 